\title{
Experimental and Numerical Investigations of Transcritical Mixing under Multi-component Gas Conditions
}

\author{
Ruitian $\mathrm{He}^{1}$, Ping $\mathrm{Yi}^{1,2^{\star}}$, Tie $\mathrm{Li}^{1,2}$, Yijie Wei ${ }^{1}$ \\ ${ }^{1}$ State Key Laboratory of Ocean Engineering, Shanghai Jiao Tong University, China \\ ${ }^{2}$ Collaborative Innovation Center for Advanced Ship and Deep-Sea Exploration, Shanghai \\ Jiao Tong University, China
}

\begin{abstract}
The mechanism of transition process from the classical two-phase to single-phase diffusioncontrolled regime has not yet been well understood, especially under the realistic multicomponent environment. In the present paper, the transcritical process is comprehensively analysed based on the experiment and molecular dynamics (MD) simulation. A singlecomponent fuel is injected into a multi-component gas mixture composed of nitrogen and combustion products at $1200 \mathrm{~K}$ and $5.25 \mathrm{MPa}$. The high-speed long-distance microscopy as well as a diode pulse laser are employed to visualize the continuous transcritical process of micrometre droplets with the gradually diminishing surface tension. Based on MD simulation, the transcritical processes in the ambient composed of pure nitrogen and multi-component gas are predicted. The simulation results reveal that the existence of carbon dioxide and water in the gas phase accelerates the transcritical process of fuel, because of the larger dissolubility of gas and the enhanced liquid temperature. In addition, it is found that the criterion based on the radial distribution function (RDF) can accurately predict the transition time, instead of the classical criterion with zero surface tension. Furthermore, this study highlights the non-ideal behaviours under diesel engine-like conditions, especially when the high polarizability of water molecule has been considered.
\end{abstract}

\section{Keywords}

Transcritical, Realistic multi-component gas, Molecular Dynamics, Experiment

\section{Introduction}

To enhance the engine performance and improve emissions, the operating pressure in diesel engines is increasingly enhanced. Since the pressure and temperature of the in-cylinder gas are much higher than the critical points of the injected fuel, it is potential for the fuel to go through the transcritical transition process. Although the supercritical mixing and subcritical phase change behaviors have been extensively analyzed, the mechanism of the transcritical transition process has not been well understood. Many key issues remain to be solved, two of which are that under what conditions the injected fuel will undergo the above-mentioned transcritical transition process, and how the transcritical behaviors be affected by the realistic multi-component ambient gas.

Many researches visualized the transcritical transition processes optically. Crua et al. [1] captured the transcritical transition processes of single-component droplets at the micrometre scale. Their results found that the droplet composition significantly affected the transcritical time and the droplet deformation, while the mixing regime boundaries is insensitive to it. Wei et al. [2] employed the long-distance microscopy and showed that the edge of the near-nozzle droplets became blurred after the injection into supercritical environment. This indicates the transition of the droplets from subcritical to supercritical state. In addition, the boundary of the supercritical sprays is observed to be totally different from that under subcritical conditions. 
However, based on the above experimental investigations, detailed information inside the sprays, such as the thermodynamic and transport properties, are difficult to be detected. Hence, the molecular dynamics (MD) simulation has been a promising tool for investigating the non-equilibrium transcritical phenomenon. Mo and Qiao [3] investigated the transcritical transition of the single-component hydrocarbon fuels from the subcritical to supercritical regime in the pure nitrogen environment. They concluded that the dimensionless transition time for the single-component system is insensitive to the liquid film thickness, but highly dependent on the ambient temperature and pressure. Nevertheless, in the above studies, the assumption of pure nitrogen environment is adopted to simplify the simulation framework. Vishnyakov et al. [4] first developed vapor-liquid equilibrium data for the blends of linear hydrocarbon, nitrogen, carbon dioxide and water, which are the main composition of the realistic ambient. Good agreement for the binary mixture of alkane and water or carbon dioxide against the experimental data was obtained. Their result also showed that their model can well predict the solubility of water in dodecane. However, in their study, only vapor-liquid equilibrium data are provided, the mechanism of the transcritical transition is limited.

The purpose of the present study is to gain an insight into the mechanism of the transcritical transition under the realistic multi-component ambient conditions based on MD simulations. The paper is organized as follows. First, the experimental arrangement and the force fields for each species are described. Then, the transcritical behavior of $n$-dodecane under pure nitrogen and multi-component ambient conditions are analyzed. Finally, major conclusions are summarized.

\section{Material and Methods}

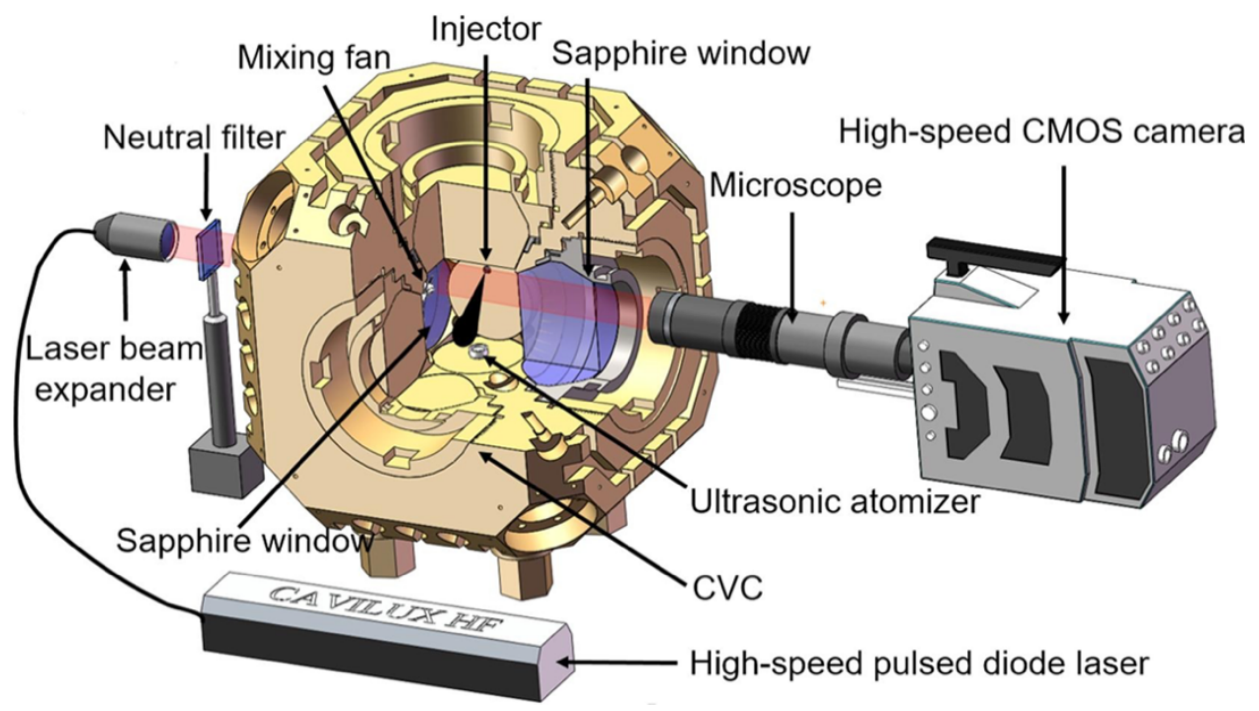

Figure 1. Schematic of the experimental arrangement

Since the experimental equipment of this study was similar to that in previous work [2], only a brief introduction will be given in this section. To visualize the droplet generated in the nearnozzle field, the microscopic imaging system was employed in this study, including the pulseddiode laser, the high-speed CMOS camera, and the long-distance microscope, as shown in Figure 1. The pulse laser beam was generated by a pulsed-diode laser (CAVILUX HF) to illuminate the droplets. To clearly capture the droplets in micrometer scale, the long microscope was configured for an optical magnification of $3.5 \times$. 


\section{Numerical Models}

\section{Molecular dynamics model}

In the present study, the transferable potentials for phase equilibria-united atom (TraPPE-UA) force field [6], the Rivera-Alejandre model [7], the EPM2 model [8], and the TIP4P-2005 model [9] have been used to simulate the $n$-dodecane, nitrogen, carbon dioxide and water molecules, respectively, as shown in Table 1. The united atom model, which groups the atom into fewer interaction sites, has been employed, because it is computationally efficient compared with the all-atom (AA) models, and its accuracy has been widely validated against the experimental data.

Table 1 - Models for $n$-dodecane, nitrogen, carbon dioxide and water molecules.

\begin{tabular}{cc}
\hline Molecule & Model \\
\hline$n$-Dodecane & TraPPE-UA force field [6] \\
Nitrogen & Rivera and Alejandre model [7] \\
Carbon dioxide & EPM2 [8] \\
Water & TIP4P-2005 [9] \\
\hline
\end{tabular}

The interatomic potential in the united atom (UA) model $\left(U_{\text {total }}\right)$ is described as

$$
U_{\text {total }}=U_{i, j}+U_{b}+U_{\theta}+U_{\varphi}
$$

where $U_{i, j}, U_{b}, U_{\theta}$ and $U_{\varphi}$ denote the non-bonded, bond stretch, bond angle bending, and bond angle torsion potentials, respectively.

The non-bonded interactions are expressed by the Lennard-Jone (LJ) 12-6 potentials and Coulombic interactions as

$$
U_{i, j}=4 \varepsilon_{i, j}\left\{\left[\left(\frac{\sigma_{i, j}}{r_{i, j}}\right)^{12}-\left(\frac{\sigma_{i, j}}{r_{i, j}}\right)^{6}\right]-\left[\left(\frac{\sigma_{i, j}}{r_{c}}\right)^{12}-\left(\frac{\sigma_{i, j}}{r_{c}}\right)^{6}\right]\right\}+\left\{\frac{q_{i} q_{j}}{4 \pi \varepsilon_{o} r_{i, j}}\right\}
$$

where $\sigma_{i, j}, \varepsilon_{i, j}, r_{i, j}, q_{i}$ are the LJ size, LJ well depth, separation and partial charge, respectively; $r_{c}$ represents the cut-off distance beyond which the potentials are negligible. When simulating the n-heptane molecules, the term of Coulombic interaction is neglected, for the reason that it is considered to be non-polar in the OPLS-UA model.

To determine the parameters for the LJ interactions between different kinds of atoms, the Lorentz-Berthelot combining rule [10] is employed as

$$
\varepsilon_{i, j}=\sqrt{\varepsilon_{i} \times \varepsilon_{j}} \sigma_{i, j}=\left(\sigma_{i}+\sigma_{j}\right) / 2
$$

A harmonic potential is adopted to describe the bond stretching as

$$
U_{b}=k_{b}\left(r_{i}-r_{c}^{e q}\right)^{2} / 2
$$

where $k_{b}$ is the stretching coefficient; $r_{i}$ and $r_{c}{ }^{e q}$ represent the bond distance and equilibrium bond distance.

The bond angle bending is described as

$$
U_{\theta}=k_{\theta}\left(\theta_{i}-\theta_{i}^{e q}\right)^{2} / 2
$$


where $k_{\theta}$ is the bending coefficient; $\theta_{i}$ and $\theta_{i}{ }^{e q}$ refer to the bending angle and equilibrium bending angle.

The torsional potential is used to restrict the dihedral rotations around the bonds

$$
U_{\varphi}=c_{1}(1+\cos \varphi) / 2+c_{2}(1-\cos 2 \varphi) / 2+c_{3}(1+\cos 3 \varphi) / 2
$$

where $\varphi$ is the dihedral angle; $c_{1}, c_{2}$ and $c_{3}$ are the Fourier coefficients.

\section{Sensitivity of atom number ratio}

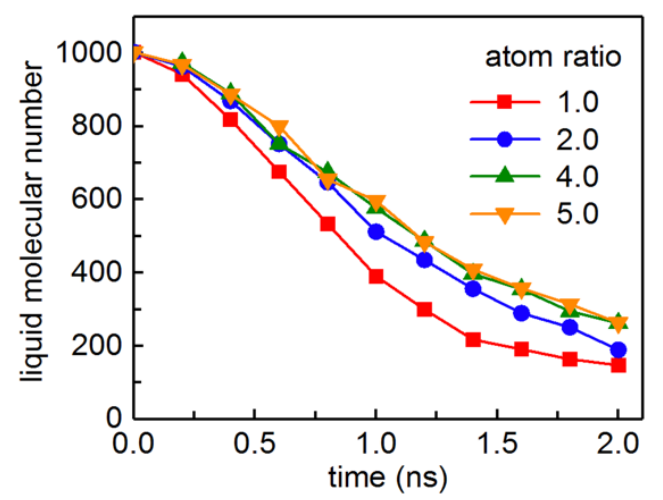

Figure 2. Effect of the ratio of atom number in gas phase to that in liquid phase on the evaporation rate of fuel;

$$
p_{a}=100 \text { bar, } T_{a}=1000 \mathrm{~K}, T_{0}=293 \mathrm{~K} \text {. }
$$

During the subcritical evaporation process, since the evaporated vapor will diffuse into the ambient, when the simulation domain is not big enough, the ambient pressure is expected to be elevated. To keep the pressure constant in the simulations, the number of gas molecules should be sufficient. Figure 2 shows the effect of ratio of atom number in gas phase to that in liquid phase on the evaporation rate of liquid film. When the ratio is larger than 4.0 , the evaporation rate becomes independent on the atom ratio. In the following studies, the ratio of 4.0 is selected.

\section{Results and Discussion}

In this section, the transcritical behaviors of the n-dodecane liquid films under various ambient conditions are investigated based on the molecular dynamics simulations. To investigate the effect of ambient gas on the transcritical transition process, the ambient gas is composed of the pure nitrogen and multi-component gas in Cases 0 and 1, respectively. The mole fractions of nitrogen, carbon dioxide and water are listed in Table 2, which are consistent with those in experiments. In both cases, the ambient pressure is set to $5.26 \mathrm{MPa}$, and the temperatures of the ambient and liquid film are 1200 and $363 \mathrm{~K}$.

Table 2 - Mole fraction of ambient gas in Cases 0 and 1, respectively.

\begin{tabular}{cc}
\hline & Mole fraction of ambient gas \\
\hline Case 0 & $x_{N_{2}}=1$ \\
Case 1 & $x_{N_{2}}: x_{\mathrm{CO}_{2}}: x_{\mathrm{H}_{2} \mathrm{O}}=21: 2: 2$ \\
\hline
\end{tabular}


Transcritical characteristics under the pure nitrogen condition
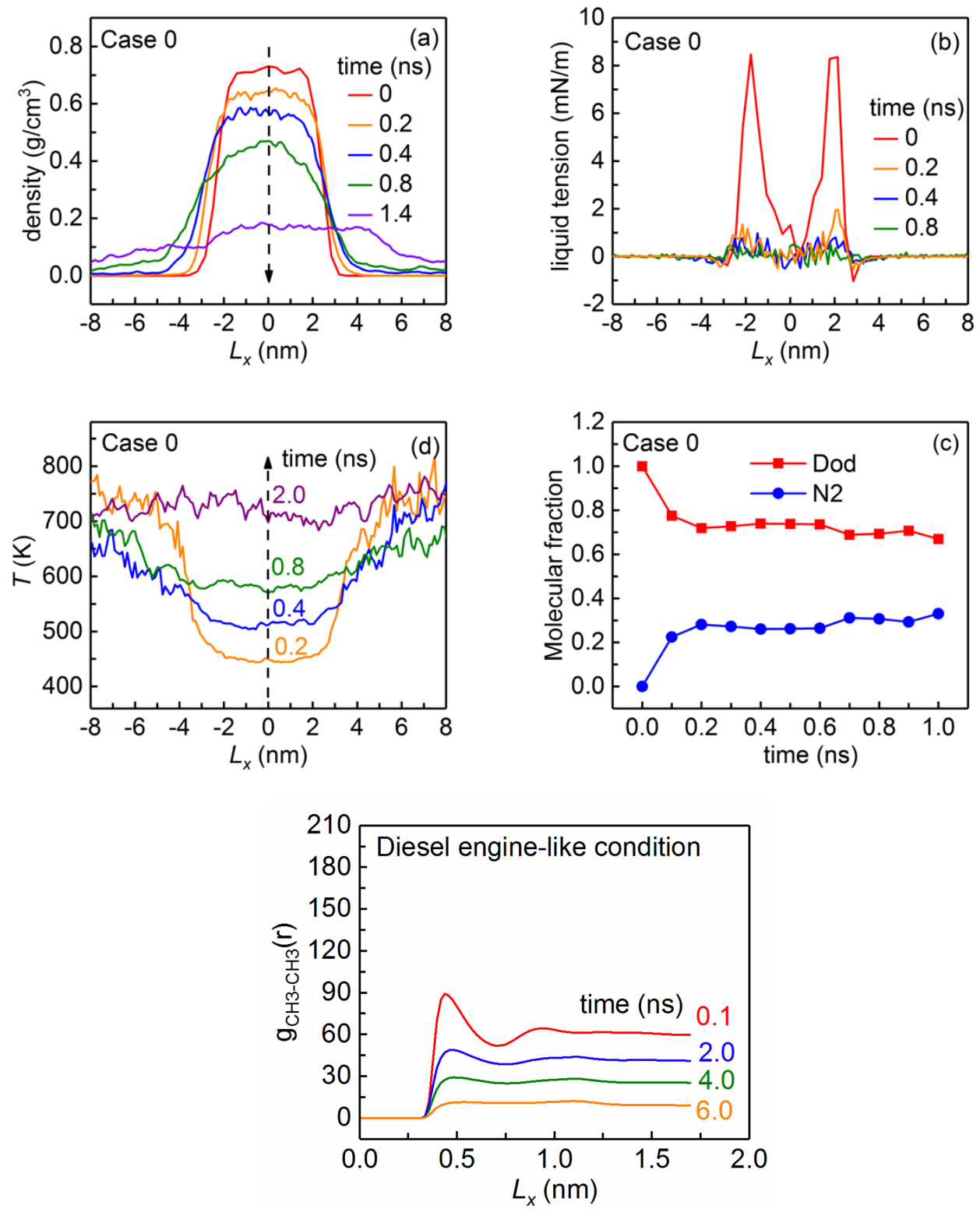

Figure 3. Spatial distribution of (a) density; (b) surface tension; (c) temperature; (d) molar fraction of fuel for the $50 \mathrm{EtOH}$ mixture at different time instants in case $1 ; p_{a}=5.26 \mathrm{bar}, T_{a}=1200 \mathrm{~K}, T_{0}=363 \mathrm{~K}$.

Since the blends of $n$-dodecane and nitrogen belongs to type III [5], its critical temperature is lower than that of $n$-dodecane, and its critical pressure is much higher than that of $n$-dodecane. However, based on the existing experimental investigations, it is difficult to detect the nitrogen dissolubility in the micrometer droplet, and evaluate the transcritical transition time accurately. Therefore, it is necessary to investigate the transcritical transition based on the molecular dynamic simulations.

As depicted from Figure 3, from 0 to $2.0 \mathrm{~ns}$, because of the rapidly increased temperature of liquid, the density of liquid film is greatly decreased from 0.68 to $0.54 \mathrm{~g} / \mathrm{cm}^{3}$. In addition, the maximum of surface tension at the vapor-liquid interface is correspondingly decreased from 15 to $4 \mathrm{mN} / \mathrm{m}$. The above phenomena indicates that even though the fuel is injected to the high-temperature and pressure environment, the fuel will not transition to supercritical regime 
immediately, but evaporates in the classical two-phase regime first. During the subcritical evaporation process, the solubility of nitrogen in the liquid is slightly increased to 0.1 at $2.0 \mathrm{~ns}$. At $4.0 \mathrm{~ns}$, it is clearly seen from Figure $3(\mathrm{a})$ and (c) that the value of surface tension is nearly vanished, but the distinct gradient of density between the ambient and fuel still exists. Based on the classical transcritical criterion it can be inferred that the transcritical transition occurs at $4.0 \mathrm{~ns}$. After $4.0 \mathrm{~ns}$, the supercritical mixing process appears.

\section{Composition effect of the ambient gas on the transcritical characteristics}

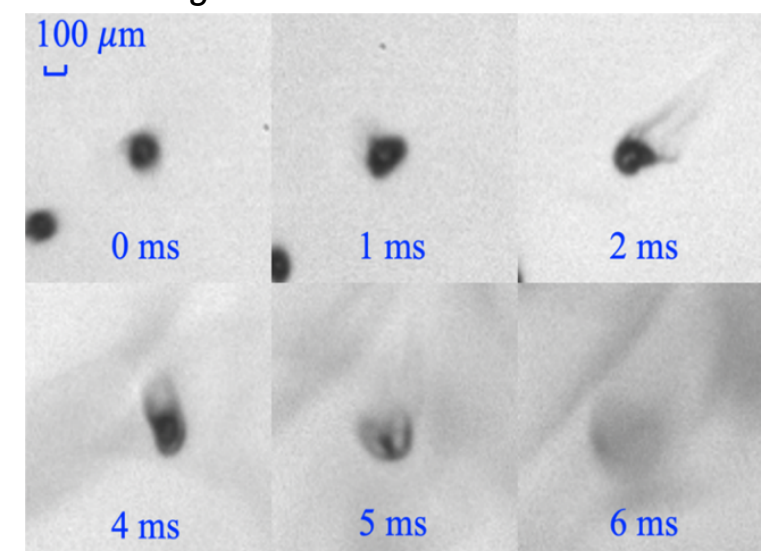

Figure 4. Images of evaporation and transcritical processes of the $n$-dodecane droplet under the multi-component ambient condition at different time instants; $p_{a}=5.26$ bar, $T_{a}=1200 \mathrm{~K}, T_{0}=363 \mathrm{~K}$.

As known, in practical combustion systems, the fuel is injected into the multi-component ambient, which consists of nitrogen and many combustion products, e.g. carbon dioxide and water. However, in most studies, the assumption of pure nitrogen environment is always adopted to simplify the simulation. To accurately analyse the multi-component effect of ambient on the transcritical transition behavior of the fuel blends, in this section, the ambient is composed of nitrogen, carbon dioxide and water, and the detailed information is listed in Table 2.

Figure 4 shows the development of the $n$-dodecane micrometre droplet, which is generated after the end of the injection. It is clearly seen that at $1 \mathrm{~ms}$, the droplet cannot maintain sphericity any more, which can be inferred that the surface tension of droplet is decreased. Then, the droplet deforms and quickly diffuses into the ambient after $2 \mathrm{~ms}$.

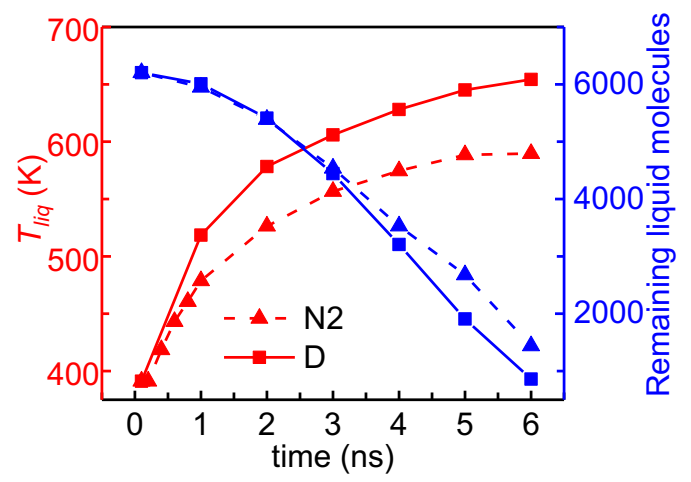

Figure 5. Temporal evolution of (a) temperature; (b) remaining molecules of fuel in the high-density fluid in Case 1; $p_{a}=$ $5.26 \mathrm{bar}, T_{a}=1200 \mathrm{~K}, T_{0}=363 \mathrm{~K}$. 
In order to deeply investigate the effect of ambient composition on the transcritical behavior, Figure 5 gives the comparison of the temperature of fuel mixture and remained fuel molecules under the pure nitrogen and multi-component gas conditions, and Figure 6 depicts the mole fraction of each species in the high-density fluid. Compared with the pure nitrogen condition in Figure 3(d), the evaporation and transcritical characteristics of fuel mixture can be very different. Firstly, the heat transfer from the ambient to fuel mixture is promoted by the existence of combustion products (i.e., carbon dioxide and water). As seen in Figure 5, at 6 ns, the temperatures of fuel mixture are 654 and $590 \mathrm{~K}$ under the pure nitrogen and the multicomponent gas conditions, respectively. This leads to the enhanced evaporation rate of fuel during the initial evaporation period. As shown in Figure 5, at $4 \mathrm{~ns}$, the number of the remained fuel molecules in the high-density fluid is decreased from 3528 to 3207 from the singlecomponent to multi-component condition. Secondly, the content of the ambient gas in the high-density fluid is elevated by the addition of combustion products in the ambient. As seen in Figures 3(d) and 4, the mole fraction of fuel in the high-density fluid is decreased from 0.8 to 0.7 at $4 \mathrm{~ns}$, when the composition of ambient is changed from the pure nitrogen to the multicomponent gas mixture. The increased content of ambient gas with high temperature also leads to the increased temperature of fuel mixture. Last but most importantly, the transcritical transition process is accelerated by the existence of combustion products. By comparing the radial distribution functions (RDF) of $\mathrm{g}_{\mathrm{CH}_{3}-\mathrm{CH}_{3}}$ in Figures 3(e) and 6(b), at $4.0 \mathrm{~ns}$, the second peak with the value of 28.3 occurs under the pure nitrogen environment, while the second peak of RDF is too weak to be captured under the multi-component ambient condition. This indicates that the high-density fluid located at the center is no longer the pure liquid phase, but the supercritical fluid with a less-ordered intermolecular structure.

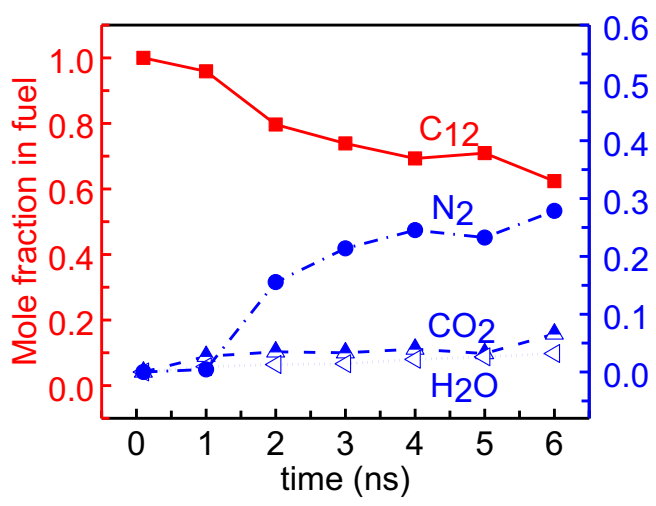

Figure 6. Temporal evolution of mole fraction in the high-density fluid in Case $1 ; p_{a}=5.26 \mathrm{bar}, T_{a}=1200 \mathrm{~K}, T_{0}=363 \mathrm{~K}$.

It should be noted that though the concentrations of carbon dioxide and water are identical in the ambient, their solubilities in the fuel mixture are different. As seen in Figure 6, at $4 \mathrm{~ns}$, the mole fraction of carbon dioxide is 0.04 , while that of water is 0.02 . The lower dissolution of water is mainly caused by the high polarizability of water molecule.

\section{Conclusions}

The experimental investigation and molecular dynamics simulations have been performed to understand transcritical behaviors under realistic multi-component ambient conditions. The major findings are 
1. The existence of carbon dioxide and water in the ambient accelerates the transcritical transition process of injected fuel, because heat transfer from the ambient to the fuel is promoted and the solubility of gas in the fuel is increased.

2. Compared with the carbon dioxide, the solubility of water is much lower, because of its high polarizability, which needs to be seriously taken into consideration in the simulations.

3. The classical transcritical criterion with the large density gradient and vanished surface tension is not sufficient to determine the transcritical time. The new criterion, based on the radial distribution function, is suggested to accurately identify the transcritical transition process.

\section{Acknowledgments}

This work was supported by the National Natural Science Foundation of China (52001209, 52020105009), Natural Science Foundation of Shanghai (20ZR1429300), and Shanghai Pujiang Program (GJ0100013).

\section{References}

[1] Crua, C., Manin, J., Pickett, L. M., 2017, Fuel, 208, pp. 535-548.

[2] Wei, Y., Li, T., Huang, S., Wang, B., Jul. 22.-26. 2018, 14th Triennial International Conference on Liquid Atomization and Spray Systems.

[3] Mo, G., and Qiao, L., 2017, Combust and Flame, 176, pp. 60-71.

[4] Vishnyakov, A., Weathers, T., Hosangadi, A., Chiew, Y.C., 2020, Fluid Phase Equilibria, $514,112553$.

[5] Clifford, A., and Clifford, T., 2002, "Fundamentals of Supercritical Fluids."

[6] Chen, B., Potoff, J. J., Siepmann, J. I., 2001, Journal of Physical Chemistry B, 105, pp. 3093-3104.

[7] Riveraa, J. L., and Alejandre, J., 2017, Colloids and Surfaces, 207(1-3), pp. 223-228.

[8] Harris, J. G., and Yung, K. H., 1995, Journal of Physical Chemistry, 99, pp. 12021-12024.

[9] Abascala, J. L. F., and Vega, C., 2005, The Journal of Chemical Physics, 123, 234505.

[10] Lorentz, H. A., 1881, Annalen der physik, 248, pp. 127-136. 